\title{
Redes sociales como factor incidente en el área social, personal y académica de alumnos de Educación Secundaria Obligatoria
}

\author{
Social networks as incidental factor on social, personal and academic lives of \\ Compulsory Secondary Education students
}

\author{
Erika González García \\ e-mail: erikag@ugr.es \\ Universidad de Granada. España \\ Nazaret Martínez Heredia \\ e-mail: nazareth@ugr.es \\ Universidad de Granada. España
}

\begin{abstract}
Resumen
Desde hace algún tiempo, las emociones han ido adquiriendo un importante papel en todas las esferas de nuestra sociedad, es por ello que numerosas investigaciones las han incluido como objeto de estudio, desde la psicología, la neurociencia, la política... también dentro de la comunicación y en concreto, relacionándolas con las redes sociales, como es nuestro caso. En este trabajo nos hemos centrado, en realizar una revisión bibliográfica acerca de las redes sociales, así como en analizar los factores sociales, personales y académicos de la incidencia de éstas en los jóvenes de Granada a través del uso las redes sociales. El artículo presenta una panorámica de algunas investigaciones y de la nuestra propia, abarcando el estudio de las redes sociales como espacios de interacción en el cual las emociones son expresadas a través de diferentes vías. Concluimos en que a través de las redes sociales se expresan ciertas emociones y afectos, así como la configuración de la identidad personal de las personas más jóvenes.
\end{abstract}

Palabras clave: emociones; redes sociales; adolescentes; Tecnologías de la Información y la Comunicación; identidad.

\begin{abstract}
Some years ago, the emotions have been acquiring an important role in all the spheres of our society. This is the reason that numerous researches have included as an object of study, from the psychology, the neuroscience, the policy... Also inside the communication and relating them to the social networks, since it is our situation. In this study, we have centred ourselves, after realizing a shallow bibliographical about social networks, as well as analyzing social, personal and academic factors of the incidence of these in young people of Granada through the use of social networks. This article presents the panoramic one of some researches and of own ours, including the study of the social networks as spaces of interaction in which the emotions are expressed across different routes. We conclude in that across the social networks certain emotions and affections express, as well as the configuration of the personal identity of the young people.
\end{abstract}

Keywords: emotions; social networks; teenagers; Information and Communication Technology; identity.

Recibido / Received: 02-10-2017

Aceptado / Accepted: 22-02-2018

Cómo referenciar este artículo / How to reference this article:

González, E., \& Martínez, N. (2018). Redes sociales como factor incidente en el área social, personal y académica de alumnos de Educación Secundaria Obligatoria. Tendencias Pedagógicas, 32, 133-146. doi: 10.15366/ tp2018.32.010 


\section{Introducción}

“Temo el día en que la tecnología sobrepase nuestra humanidad. El mundo solo tendrá una generación de idiotas" (Einstein)

En este estudio se presenta el análisis y los resultados de un trabajo fruto de un proyecto llevado a cabo durante el curso 2016/2017 con el alumnado de Educación Secundaria Obligatoria de algunos Institutos de Educación Secundaria de la provincia de Granada, España.

En el informe de investigación que presentamos, convergen dos vías: por un lado la relación que existe entre las emociones y las redes sociales y, por otro, el estudio empírico realizado a través de un cuestionario sobre la temática que nos ocupa.

Dentro de este marco teórico consideramos fundamental conocer qué son las emociones y su relación con respecto a las redes sociales. Así pues, las redes sociales se han convertido en los nuevos espacios virtuales en los que nos relacionamos y en los que cimentamos no solo nuestra identidad. También funcionan a modo de sistema de filtro y de alerta en la medida en que permiten que ajustemos la cantidad de información que recibimos en función de nuestros propios intereses y preferencias y de los de aquellos en quienes, a primera vista, confiamos. Como argumenta Orihuela: "El sentido común y la prudencia siguen siendo los mejores consejeros en lo que respecta a la difusión de información personal en una red pública, como es Internet. Si estás en Internet y tienes un secreto, es mejor que ni lo pienses" $(2008,59)$. El fuerte impacto de las redes sociales ha calado de forma tan profunda en la población adolescente que se ha implantado un nuevo modelo de relación. Esto ha llevado a que se adopte un nuevo modelo de comunicación virtual donde la presencia física no es necesaria. Las redes sociales se han convertido en un medio de comunicación instantáneo y eficaz, mediante el cual aumenta la participación de la población de un modo social, cívico y político (García, Seco y Del Hoyo, 2013). En este sentido, nos vemos afectados por el uso de internet en todos los ámbitos de nuestras vidas. Dado este efecto, se ha incorporado su estudio a las numerosas ramas del saber así como la influencia que poseen ante las emociones.

Una vez elaborado el marco teórico, aludiremos el estudio empírico desarrollando una metodología cuantitativa, que ha consistido en la realización de un cuestionario adaptado de la Universidad del Azuay para conocer el poder que poseen las redes sociales sobre las emociones de los alumnos de Educación Secundaria Obligatoria de Granada.

Finalmente, extraeremos algunos resultados y conclusiones teniendo presente el objetivo del que partíamos al inicio de este estudio.

\section{Marco referencial: en torno a las emociones y a las redes sociales}

La diversidad de sustratos y manifestaciones de las emociones complica bastante la elaboración de una definición comprehensiva. No obstante, consideramos que constituyen un elemento esencial de nuestra existencia, pues marcan nuestras reacciones frente al medio e influyen en nuestras percepciones. Las investigaciones sobre emociones y sentimientos se han llevado a cabo desde una amplia variedad de disciplinas, como la psicología, la neurociencia, la educación, la sociología, etc.

Es muy común que cuando hablamos de emociones y sentimientos confundamos dichos conceptos, ya que estos términos están estrechamente relacionados y producen una respuesta semejante en la persona que los experimenta.

Puesto que las emociones están asociadas con el temperamento, la personalidad y la motivación de las personas, éstas influyen de distinta forma a unos sujetos y a otros, promoviendo un tipo de respuesta u otra. La liberación de hormonas y neurotransmisores causan los estados emocionales, y más tarde, la transformación de dichas emociones en sentimientos, en algunos casos. A modo general, hay evidencias de que las emociones son consideradas de menor duración que los sentimientos, aunque de mayor intensidad. Las emociones no siempre pueden ser controladas, 
puesto que muchas pueden crear impulsos.

Las emociones han estado presentes en el hombre desde su aparición. Posiblemente la primera teoría enunciada en este sentido fue la de Platón, que se aproxima a la emoción denominada miedo, al establecer que se produce dolor cuando la proporción o la armonía de los elementos que componen al ser vivo, es amenazada o comprometida, y se produce placer cuando tal proporción o armonía es restablecida.

El diccionario de la Real Academia Española de la Lengua, en su primera acepción, considera que una emoción no sólo contiene una respuesta corporal, sino que es una "alteración del ánimo intensa y pasajera, agradable o penosa, que va acompañada de cierta conmoción somática" (RAE, 2017).

Las emociones constituyen el sistema motivacional humano, determinando y organizando la conducta, y pueden tener efectos favorables o desfavorables en la salud de las personas (Garrido, 2006).

Tienen su origen biológico en un conjunto de estructuras nerviosas denominadas sistema límbico, que incluyen el hipocampo, la circunvolución del cuerpo calloso, el tálamo anterior y la amígdala. Esta última además de desempeñar otras funciones es la gestora principal de las emociones, y cualquier lesión que pueda sufrir anula la capacidad emocional (Ledoux, 1996).

A pesar de que todas las personas a lo largo de la vida hemos experimentado emociones, nos resulta muy complicado definir de manera clara lo que es una emoción, "las emociones se viven, se sienten, se reconocen, pero solo una parte de ellas se puede expresar con palabras" (Esquivel, 2001, 10).

Siguiendo a Esquivel, (2001), existen varios factores que dificultan poder llegar a una definición concreta de las emociones, algunos de ellos son:

- El factor subjetivo que estas provocan, por ejemplo: cuando le preguntamos a distintas madres acerca del sentido al dar a luz estas darán respuestas y sentimientos distintos como pueden ser alegría, miedo, dolor, curiosidad.

- Otro factor es la imposibilidad de conocer todas las emociones que existen, puesto que son diferentes en cada persona y son muy numerosas, cada vez que pensamos o nos relacionamos surgen diversas emociones que es imposible recordar totalmente y mucho menos ser capaces de explicar a otras personas todo lo que te produce aquello que vives, por lo que nunca podremos llegar a conocer de forma total todas las emociones.

De acuerdo con Camps (2012) la definición de emoción es algo más complejo de lo que en un primer momento parece. En la vida cotidiana manejamos términos como emoción, sentimientos, pasión y otras muchas palabras similares para expresar estados de excitación que nos producen alteraciones físicas, como sudor, palpitaciones, dolores musculares, temblores... estados psicológicos de bienestar o malestar, así como pensamientos y conductas relacionados con estos estados. En este sentido y siguiendo a Camps (2012), una emoción tiene que cumplir cuatro criterios para considerarse como tal: 1- La emoción tiene una connotación o significado específico y subjetivo único para cada persona. 2- Va acompañada de una expresión facial determinada y muy concreta. 3- Tiene un patrón de descarga neuronal específico, es decir, unas reacciones físicas singulares y determinadas. 4- Cada emoción origina unos comportamientos concretos que podemos reconocer como propios de ella.

Oakley y Jenkins (1992), Reeve (1994), Casacuberta (2000), Bisquerra (2000 y 2011) y Morgado (2007), piensan que en toda emoción el sujeto evalúa un estímulo, consciente o inconscientemente, en función de que resulte relevante para alcanzar un objetivo. Para ellos, es muy importante no sólo la evaluación sino el estado y los cambios corporales y psíquicos que pueden producir en los sujetos en función de que se alcancen o no los objetivos propuestos, dando lugar a emociones positivas o 
negativas. Las emociones se sienten, es decir, no son un puro proceso de razonamiento. En este sentido, pensamos que el estado emocional de una persona determina la forma en que percibimos el mundo.

Las emociones tienen un componente cultural y psicológico. Hay tantas variaciones emocionales como culturas, por esta razón no es posible relatar la historia de una emoción. No son objetos, sino que se representan para hacerlas significativas a través de una serie de parámetros culturales. Se pueden experimentar, pero también provocar facilitándole a los sujetos unos estímulos convincentes, como suele suceder en la publicidad que utilizan los medios de comunicación (Tausiet y Amelang, 2009). En esta misma línea, Gergen (2015), argumenta que no podemos dudar de que sintamos emociones, pues éstas están ubicadas en la mente y podemos identificarlas fácilmente. "Son formas de acción que adquieren su inteligibilidad en las relaciones y obtienen su valor a partir del uso social que de ellas se hace" (Gergen, 2015, 167). Las emociones salen a la luz cuando las experimentamos, es decir, a través de las palabras, de los gestos e incluso de rasgos fisiológicos que se activan. Según la circunstancia en la que nos encontremos, la respuesta a la situación emocional puede variar, pues ésta puede ser más compleja y amplia.

Otros autores definen la emoción como "una respuesta o reacción ante un estímulo significativo que el sujeto evalúa, consciente o inconscientemente, en función de sus objetivos o intereses e influido por su ideología y por el contexto sociocultural”' (Beas, González y Salmerón, 2016, 54).

Desde el punto de vista del relativismo cultural, la vida emocional ha desarrollado interesantes trabajos en las últimas décadas. La idea de que nuestras emociones dependen del lugar y del momento histórico que nos ha tocado vivir, ha dado origen a una nueva ciencia, la emocionología, en estrecha relación con otras ciencias como la sociología o la psicología. Según Peter Stearns, autor que acuñó este término, "la emocionología de una sociedad estudia las normas de expresión y ocultación de las emociones que de manera explícita o implícita regulan la vida cotidiana de los individuos que pertenecen a la misma" (Stearns y Stearns, 1985, 823). En síntesis, la presencia y el contenido emocional del significado de la vida social no se pone en duda. Como argumenta Mahamud:

"La clave está en qué significado afectivo otorgamos a los diversos y múltiples componentes de nuestras vidas $[\ldots]$ se comprende así que exista un mundo emocional, que los objetos, conceptos y fenómenos adquieran y posean un significado emocional: patria, religión, maternidad, madre, familia, hogar, constituyen una serie de elementos cargados de emociones intensas, llenas de significado y valor." (2012, 20)

Consideramos que las emociones son un proceso a través del cual un estímulo provoca en las personas unas determinadas reacciones, las cuales son cambiantes, dependiendo de numerosos factores. Para Carrascal:

"Todas las personas, tanto niños como adultos, tenemos emociones, pero ¿realmente sabemos lo que significan? O ¿lo que producen en nosotros? La mayoría del tiempo tenemos emociones y muchas veces no somos conscientes de ello o simplemente no sabemos distinguirlas. Las emociones se refieren al modo de sentirnos en el mundo, que están referidas a objetos. Más aún, el objeto de las emociones está investido de valor o importancia para las personas. Las personas según las diferentes situaciones en las que tienen que desenvolverse expresarán una emoción u otra. Por ejemplo, si estás jugando con tus amigos te sentirás feliz en cambio si te roban sentirás tristeza e ira." (2016, p. 87).

Como podemos comprobar, dar una definición no es cuestión de todo o nada, pues para lo que a un investigador le es interesante, para otro es todo lo contrario.

En general, podemos deducir que la mayoría de los autores investigados consideran que toda emoción consta de tres componentes básicos: cognitivo, evaluativo y apetitivo.

En cuanto a los tipos que existen de emociones, hemos comprobado que a pesar de la multitud de clasificaciones que se han hecho: emociones, simples, básicas, primarias, secundarias, sociales, retrospectivas, ambigua, etc. La mayoría de investigadores se posicionan en dos tipos 
principalmente, como son las emociones positivas y las emociones negativas. En este sentido, las emociones se generan a partir de un acontecimiento que de alguna manera puede afectar a nuestro bienestar. En función de cómo pueda afectarnos ese acontecimiento, la emoción puede ser negativa o positiva, nos provoca bienestar o malestar. Por ejemplo, el miedo puede ser una emoción negativa porque nos provoca malestar, pero sería positiva si ese miedo nos ayudara a ser prudentes ante ciertos acontecimientos.

Una vez aclarado el concepto de emoción, procederemos a definir de forma breve el concepto de red social y la relación que existe con respecto a las emociones.

El término red social está directamente relacionado con la integración de las tecnologías, tecnologías que están presentes en nuestra vida y que nos acompañan en cada momento, ya que nos hemos convertido en los principales actores/consumidores de esta oportunidad surgida en el seno de Internet. Se convierte así, en un escenario que, en ocasiones, puede generar riesgos para una generación que se encuentra en una etapa de crecimiento personal, emocional, social... En este sentido, es necesario construir las herramientas adecuadas para poder navegar por este entramado que puede presentar riesgos, sobre todo entre la población más joven (Llamas y Pagador, 2014).

En sentido amplio, las redes sociales pueden definirse "como una estructura social formada por personas o entidades conectadas y unidas entre sí por algún tipo de relación o interés común" (Ponce, 2012, 2). En la actualidad, intercambiar información se está convirtiendo en algo habitual, la sociedad está sufriendo cambios acelerados, en parte, debido al gran avance de las Tecnologías de la Información y la Comunicación y a la globalización (De Haro, 2010). Así pues nos definen y acompañan, formando ya una parte fundamental de nuestras vidas, viendo como muchas veces se nos hace inimaginable el hecho de no llevar un smartphone en nuestro bolsillo. Este hecho ha dado lugar a que dependamos de ellas hasta el punto en que las utilicemos por obligación y necesidad en lugar de entretenimiento en el tiempo libre, convirtiéndonos en verdaderos dependientes de las mismas, influyendo éstas incluso en nuestro bienestar. Es tanta su importancia para nosotros, que aquellos que no pertenecen a alguna red social o les faltan, no se sienten parte realmente de la sociedad, es decir, no se ven totalmente integrados, ya que no están en contacto con otras personas de manera virtual, no saben de la vida de ellas y, sobre todo, no pueden mostrarse como quieren que los vean realmente.

Las redes sociales han venido a desvanecer barreras físicas, geográficas y sociales, permitiendo conectar a personas de todo el planeta. Esto constituye un logro de la tecnología, que permite que individuos que tengan un interés común, puedan darse cita y conocerse en un espacio virtual (Lampe, Ellison y Steinfield, 2006). De este modo, las personas pueden estar conectadas sin importar su ubicación.

Una vez definidos ambos términos, somos conscientes de que lo que hace tener emociones diferentes a cada persona son sus experiencias, que son el catalizador de la perspectiva de las personas frente a cualquier evento. Según un estudio llevado a cabo por Fowler y Christakis (2008), se aprecia que las emociones se contagian a los otros usuarios dependiendo de la relación y la cercanía entre ellos. Lo que significa que si una persona se relaciona con otro colectivo mayormente feliz, es mucho más probable que esa persona se contagie de esa emoción y sea más feliz, y viceversa. Este hecho es realmente importante, ya que sabiendo esto, se pueden manipular las emociones de una persona dependiendo del entorno en el que se le sitúe, o la información que éste vea a través de las redes sociales. Así pues, podemos afirmar que las redes sociales afectan de una manera muy contundente a nuestras emociones y cómo percibimos el mundo o experimentamos nuestras relaciones, dependiendo de algo tan simple como ser amigo de una u otra persona en una red social.

Al hilo de lo anterior, ¿`se forman nuevas emociones a partir del uso de las redes sociales? la respuesta hallada en numerosos artículos es afirmativa. Existen multitud de redes sociales, las cuales dan opción a expresar las emociones que te transmiten en cada momento, en cada una de las publicaciones que ves a lo largo del día. Respecto a Facebook, te da opción a poner "me gusta", 
"me entristece", "me encanta", "me enfada" o "me divierte", ya sea en noticias, en fotografías, en vídeos o en comentarios. Otra de ellas es WhatsApp, la cual posee alrededor de 250 emojis diferentes ordenados por secciones, a través de los cuales puedes manifestar tus emociones al mantener una conversación, o también puedes expresar tus emociones al recibir una fotografía, vídeo o cualquier otro documento. Es increíble como con un único emoji, la persona con la que estás hablando es capaz de identificar la emoción que te ha transmitido el mensaje que has recibido. Muchas personas son capaces de mantener una conversación únicamente con emojis. No obstante, en ocasiones, intentamos indagar lo que nos quiere transmitir, ya que cada persona puede descifrarlo de una manera diferente (Salas, 2016).

En este sentido, la información es la materia prima más ansiada, sobre todo cuando se trata del colectivo adolescente. Las redes sociales incitan a sus usuarios a mostrar la mayor cantidad de información posible así como agregar al mayor número de contactos posibles. Al mismo tiempo toda la información que nosotros añadimos en nuestros perfiles o espacios puede ayudar para captar a nuevos participantes. Este entramado de información, contactos... podríamos compararlo con una telaraña en la que se va captando información que es útil para la plataforma y que cada vez es más y más grande (Llamas y Pagador, 2014).

En el ámbito digital, el parámetro emocional está en relación al estado de identidad de cada persona. En las redes sociales cabe señalar en procesos de reconocimiento y negociaciones del status pues, como apunta Svensson (2014), cuanto más te enlaza la gente, más le da a "me gusta" en tus publicaciones, más las comentan, etc., más alto apareces en los rankings de listas de lecturas recomendadas, en los flujos de noticias de las redes sociales. Ese incremento en el estatus va unido a emociones y sentimientos de satisfacción y de bienestar. Más aún, las emociones positivas afloran cuando los usuarios son capaces de reafirmar su autoconcepto del yo. En este caso, las emociones son usadas para mejorar y ayudar en el trabajo de los usuarios en el mundo digital. Muchas personas viven conectadas a las redes sociales, esperando la reacción de sus seguidores. Una reacción que produzca un "abanico" de emociones satisfactorias y bienestar en sí mismo. Un simple "me gusta", puede provocar numerosas emociones y sentimientos en el dueño de la publicación, el cual, provocará que se sienta más popular.

Las emociones en las redes sociales las tenemos muy presentes ya que se encargan de crear emoticonos, donde los consumidores pueden expresar con libertad su estado de reacción en un momento dado. Incluso, podemos transmitir emociones y sentimientos que no sintamos sin que nadie se dé cuenta. Sabiendo que los estados emocionales se pueden contagiar a otros mediante el contacto y la cercanía, haciendo que la gente tienda a sentir emociones similares a gente de su entorno, ¿cuánto nos afecta emocionalmente ese contacto indirecto que tenemos mediante una red social? Hay estudios los cuales demuestran que las emociones en grupo son mucho más sencillas de contagiar y no solo en grupos sino también en espacios online y redes sociales. Por otro lado, también se puede ver que no solo es el contacto directo entre personas lo que hace que nuestras emociones cambien, también un mensaje indirecto en una red social como un estado o una foto hará que una persona cambie su humor o emoción frente a esa situación (Fowler y Christakis, 2008). De acuerdo con Serrano-Puche:

"Dos de las cuestiones teóricas de fondo que marcan el debate en torno a las emociones en la esfera online, tanto en los estudios en Comunicación como en disciplinas afines, son: por un lado, cómo las emociones afloran y pueden medirse en Internet (Küster y Kappas, 2014) y, por otro, las diferencias y similitudes entre la expresión de las emociones en las relaciones cara a cara y en las relaciones mediadas por la tecnología digital (Boyns y Loprieno, 2014).” (2016, 22)

Se puede observar, y más aún si te has comunicado alguna vez por redes sociales con otro usuario, que las emociones recibidas mediante la tecnología digital y las transmitidas en las relaciones cara a cara, son diferentes, aunque se pueden poner cosas en común. A priori, se podría pensar que las relaciones a través del ámbito digital son más frías por la ausencia de presencia, y esto produciría una cierta dificultad para poder expresarse, pero no es así. 


\section{Objetivo de la investigación}

Analizar el área social (equipamientos y usos de internet y redes sociales) área personal (importancia de estar conectado, evadirse de los problemas y rasgos de personalidad con o sin el uso de redes sociales) y área académica (incidencia positiva o negativa en las calificaciones obtenidas) de alumnos de educación secundaria obligatoria.

\section{Metodología de la investigación}

Para analizar qué emociones y cómo éstas afectan al día a día en los jóvenes de Granada a través del uso las redes sociales se consideró oportuno el desarrollo de una investigación de corte cuantitativo que exterioriza su finalidad epistemológica a través de un estudio descriptivo, exploratorio y trasversal, pues nos centraremos en conocer las emociones que se generan a través del uso de las redes sociales en nuestros adolescentes.

Bajo estas premisas, la población objeto de estudio está compuesta por 200 alumnos y alumnas de Educación Secundaria Obligatoria de distintos centros públicos educativos de la ciudad de Granada. El muestro se caracteriza por ser no probabilístico, sino intencional. Tras la selección de los distintos centros, se procedió a la realización del test llevado a cabo durante el segundo cuatrimestre del curso académico 2016-2017 con los permisos y autorizaciones necesarios para el desarrollo de dicha investigación. La recogida de datos fue llevada a cabo por dos profesoras de la Facultad de Ciencias de la Educación de la Universidad de Granada, las cuales forman parte del equipo de trabajo para desarrollar esta investigación. Una vez obtenidos los permisos para poder pasar el cuestionario, la realización de éste se desarrolló en una sesión, en la cual estaban presentes las dos profesionales, así como el tutor de cada aula.

La selección de la muestra posee los siguientes criterios de participación: (1) Ser estudiante de Educación Secundaria Obligatoria de la ciudad de Granada. (2) Poseer una edad de entre los 16 y 18 años. (3) Poder participar de manera voluntaria teniendo en cuenta los permisos y autorizaciones de padres, profesores y centros educativos. Atendiendo a la muestra (Tabla 1) 74 eran hombres y 126 mujeres

Tabla 1

Participantes por género

\begin{tabular}{llll}
\hline & & Frecuencia & Porcentaje \\
\hline Válidos & Hombre & 74 & 37 \\
& Mujer & 126 & 63 \\
& Total & 200 & 100 \\
\hline
\end{tabular}

Fuente: elaboración propia.

La edad media es de 17 años, siendo la moda de edad aquellos sujetos que tienen 18 años. Aunque el intervalo de edades oscila entre los 16 y 18 años.

\section{Instrumentos de recogida de información}

Para la recogida de información se empleó un cuestionario compuesto de 33 ítems, donde se combinaba preguntas de escala dicotómica y de escala likert de 1 a 5 (1: Siempre; 2: Casi siempre; 3: A veces; 4: Casi nunca; 5: Nunca), siendo estas las que predominan en todo el instrumento. El instrumento fue adaptado del desarrollado por Molina y Toledo (2014) desarrollado en la ciudad de Cuenca (Ecuador) con alumnos del primer año de Bachillerato para analizar la influencia de las redes sociales en el área personal, social, familiar y académica de los adolescentes. Aunque dicho instrumento ya poseía sus índices de fiabilidad y validez, en nuestro estudio optamos por calcularla a través del paquete estadístico SPSS versión 23.0 para Windows mediante el alfa de Cronbach, obteniendo un valor de 0,866 . 
Por último, señalar que el cuestionario se estructuró de la siguiente manera: datos demográficos (edad, sexo, curso y colegio), área social (4 preguntas), área personal (18 preguntas), área académica ( 2 preguntas) y área familiar (5 preguntas).

\section{Análisis de los datos}

El tratamiento de los datos obtenidos se efectuó a través del programa SPSS versión 23.0, referidas tanto a los objetivos como a la metodología de nuestra investigación, todos los datos han sido tratados de manera descriptiva utilizando las medidas centrales, como son los porcentajes, la media y las desviaciones típicas con el fin de conocer los datos recabados.

\section{Resultados}

A continuación, presentamos los resultados de los análisis descriptivos obtenidos a partir de la aplicación del cuestionario descrito anteriormente.

Como hemos explicado anteriormente la muestra consta de 74 hombres y 126 mujeres, siendo la edad media es de 17 años, siendo la moda de edad aquellos sujetos que tienen 18 años. Aunque el intervalo de edades oscila entre los 16 y 18 años.

\section{1. Área social}

En este apartado, abordamos aspectos relacionados con equipamientos y usos de internet, redes sociales que utilizan y uso determinado de dichas redes sociales.

En cuanto a los equipamientos y usos de internet, destacamos un mayor uso del móvil (Smartphone) con un $97,5 \%$, seguido del ordenador con un $2,5 \%$.

Tabla 2

Equipamientos TIC

\begin{tabular}{ccc}
\hline & & Porcentaje válido \\
\hline Válidos & Móvil & 97,5 \\
& Ordenador & 2,5 \\
& Total & 100,0 \\
\hline
\end{tabular}

Fuente: elaboración propia.

Atendiendo al uso que se realiza de Internet prima para el entretenimiento $(29,8 \%)$ y la conexión en las redes sociales $(21,7 \%)$, seguido hacer amigos con un $18,6 \%$. Finalmente hacen uso para jugar y una manera de pasar el tiempo con un 14,9\%.

Tabla 3

Usos de Internet

\begin{tabular}{llll}
\hline & & Frecuencia & Porcentaje válido \\
\hline Válidos & Amigos & 30 & 18,6 \\
& Entretenimiento & 48 & 29,8 \\
Paso del tiempo & 24 & 14,9 \\
Jugar & 24 & 14,9 \\
Redes Sociales & 35 & 21,7 \\
Total & 161 & 100,0 \\
\hline
\end{tabular}

Fuente: elaboración propia.

En cuanto a las redes sociales que frecuentan, podemos ver un uso elevado de Facebook con un $65 \%$, seguido de Instagram (23,5\%), Twitter (9\%) y YouTube $(2,5 \%)$. 
Tabla 4

Tipo de redes sociales que utilizan

\begin{tabular}{llll}
\hline & & Frecuencia & Porcentaje válido \\
\hline Válidos & Facebook & 130 & 65,0 \\
& Twitter & 18 & 9,0 \\
& Instagram & 47 & 23,5 \\
& YouTube & 5 & 2,5 \\
& Total & 200 & 100,0 \\
\hline
\end{tabular}

Fuente: elaboración propia.

Finalmente, dentro del área social destacamos un mayor uso de las redes sociales para hacer amigos con un porcentaje del 35,4. Del mismo modo, para conocer páginas de moda $(32,8 \%)$ y la curiosidad de ver otros perfiles $(19,7 \%)$, así como la comunicación con gente del extranjero $(9,6 \%)$ y por último existe un menos uso de las redes sociales para conocer nuevos amigos con un 2,5\%.

Tabla 5

Uso de redes sociales

\begin{tabular}{llll}
\hline & Frecuencia & Porcentaje válido \\
\hline Válidos & Hacer amigos & 70 & 35,4 \\
& Comunicación al extranjero & 19 & 9,6 \\
Moda & 65 & 32,8 \\
Curiosidad & 40 & 19,7 \\
Conocer gente & 6 & 2,5 \\
Total & 200 & 100,0 \\
\hline
\end{tabular}

Fuente: elaboración propia.

Una vez más observamos la importancia del uso del móvil dentro de los jóvenes de la ciudad de Granada para su entretenimiento personal así como conocimiento de nuevas personas, y uso de las principales redes sociales, es decir, Facebook e Instagram.

\subsection{Area personal}

En este apartado haremos especial mención a 4 preguntas, ya que creemos de importante relevancia. Éstas hacen referencia, a la importancia de estar conectado frecuentemente a las redes sociales, la importancia que poseen para olvidarse de los problemas, por último el rasgo de personalidad con el que se identifican con y sin el uso de las redes sociales.

En relación a la importancia del uso constante de las redes sociales vemos que no es muy importante estar constantemente conectado con un $61 \%$.

Tabla 6

Uso de redes sociales

\begin{tabular}{llll}
\hline & & Frecuencia & Porcentaje válido \\
\hline Válidos & Sí & 78 & 39,0 \\
& No & 122 & 61,0 \\
& Total & 200 & 100,0 \\
\hline
\end{tabular}

Fuente: elaboración propia.

En cambio existe una importancia del uso de las redes sociales para evadirse de los problemas con un $64,6 \%$ de respuesta positiva. 
Tabla 7

Importancia de las redes sociales para evadirse de los problemas

\begin{tabular}{llll}
\hline & & Frecuencia & Porcentaje válido \\
\hline Válidos & Sí & 130 & 64,6 \\
& No & 70 & 35,4 \\
& Total & 200 & 100,0
\end{tabular}

Fuente: elaboración propia.

Haciendo referencia a los rasgos de personalidad con los que se identifican sin el uso de las redes sociales los alumnos han respondido con un $43 \%$ que son sociales, tranquilos $(24,5 \%)$, tímidos $(21,5 \%)$, extrovertidos $(6 \%)$ y por último atrevidos $(5 \%)$.

Tabla 8

Rasgos de personalidad con el que se identifican

\begin{tabular}{clll}
\multicolumn{4}{c}{ Rasgos de personalidad con el que se identifican } \\
\hline \multirow{4}{*}{ Válidos } & Trecuencia & Porcentaje válido \\
\cline { 2 - 3 } & Tranquilo & 43 & 21,5 \\
& Extrovertido & 12 & 24,5 \\
& Sociable & 86 & 6,0 \\
& Total & 200 & 43,0 \\
\hline
\end{tabular}

Fuente: elaboración propia.

Por el contrario, si contrastamos los datos anteriores con los datos obtenidos atendiendo a los rasgos de personalidad con el que se identifican, pero cuando usan las redes sociales, vemos que ser sociales sigue siendo el principal con un $34,5 \%$, seguido de tranquilos con un $24 \%$, tímido (17\%), atrevido $(12,5 \%)$ y extrovertido (12\%). Existe un cambio de personalidad con el uso de las redes sociales, con su uso se vuelven más atrevidos y menos extrovertidos.

Tabla 9

Rasgos de personalidad con el que se identifican cuando usan las redes sociales

\begin{tabular}{llll}
\hline & & Frecuencia & Porcentaje válido \\
\hline Válidos & Tímido & 34 & 17,0 \\
& Tranquilo & 48 & 24,0 \\
& Extrovertido & 24 & 12,0 \\
Sociable & 69 & 34,5 \\
& Total & 200 & 100,0
\end{tabular}

Fuente: elaboración propia.

Sin duda alguna, la alta frecuencia del uso de las redes sociales supone un cambio en los rasgos de personalidad en nuestros adolescentes.

\section{3. Área académica}

En el área académica nos centramos en conocer la influencia positiva o negativa de las redes sociales, en las calificaciones obtenidas por el alumnado. En ambas preguntas (creencia sobre su influencia negativa en sus estudios y la bajada en las calificaciones por el uso de las redes sociales) vemos un alto porcentaje positivo $62,5 \%$ y $63,5 \%$ respectivamente, por lo que debemos tener en cuenta que las redes sociales son un fuerte factor de impacto en las calificaciones de nuestros alumnos. 
Tabla 10

Importancia de las redes sociales para evadirse de los problemas

\begin{tabular}{|c|c|c|c|c|}
\hline & & & $\begin{array}{l}\text { Crees que influyen } \\
\text { negativamente }\end{array}$ & Han bajado tus notas \\
\hline & & Frecuencia & Porcentaje válido & Porcentaje válido \\
\hline \multirow[t]{3}{*}{ Válidos } & Sí & 103 & 62,5 & 63,5 \\
\hline & No & 77 & 38,5 & 36,5 \\
\hline & Total & 200 & 100,0 & 100,0 \\
\hline
\end{tabular}

Fuente: elaboración propia.

\section{4. Área familiar}

Por último, dentro del área familiar destacaremos el distanciamiento hacia familiares y amigos por el uso de las redes sociales, así como las reacciones ante un problema grave ocurrido en las redes sociales.

Atendiendo al distanciamiento provocado por el uso de las redes sociales, vemos como los alumnos afirman con un $85,5 \%$ la existencia de un desapego hacia familiares y amigos.

Tabla 11

Distanciamiento por el uso de las redes sociales

\begin{tabular}{|c|c|c|c|}
\hline & & Frecuencia & Porcentaje válido \\
\hline \multirow[t]{3}{*}{ Válidos } & Sí & 49 & 85,5 \\
\hline & No & 147 & 24,5 \\
\hline & Total & 200 & 100,0 \\
\hline
\end{tabular}

Fuente: elaboración propia.

Por otro lado, observamos que la principal reacción ante problemas graves ocurridos por el uso de las redes son cerrar la puerta con un 24,5\%, avisar a los padres con un $24 \%$ pero no a los amigos (5\%), denunciar también posee un elevado porcentaje, un 19,5\%. En cambio, tener cuidado y continuar junto con no avisar no suele ocurrir de manera frecuente.

Tabla 12

Reacción ante problemas graves ocurridos en las redes sociales

\begin{tabular}{llll}
\hline \multicolumn{3}{c}{ Reaccion ante problemas graves ocurridos en las redes sociales } \\
\hline Válidos & Cerrar la puerta & 49 & 24,5 \\
& Avisar a mis padres & 48 & 24,0 \\
& Avisar a un amigo & 10 & 5,0 \\
& Denunciar & 39 & 19,5 \\
Pelear & 25 & 12,5 \\
No avisar & 5 & 2,5 \\
Total & 200 & 100,0
\end{tabular}

Fuente: elaboración propia.

\section{Discusión y conclusiones}

A través de la realización de este trabajo hemos pretendido indagar sobre una realidad esencial en nuestra sociedad, como es el caso de las emociones de nuestros adolescentes, empleando para ello diversos Institutos de Educación Secundaria Obligatoria de la ciudad de Granada. A modo de conclusión y tal como venimos argumentando a lo largo de este trabajo, estamos inmersos en la sociedad de la información por medio de las tecnologías, como son las redes sociales, pero la 
información de la que disponemos, muy pocas veces sabemos aplicarla de manera práctica a nuestras vidas. No cabe duda que hoy en día quien no hace uso de las redes sociales no está a la "moda", sin embargo, éstas tienen sus ventajas y desventajas y casi todo el mundo hace uso de ellas aun sin saber el riesgo que conllevan en muchas ocasiones. Las redes sociales han logrado convertirse en una "Revolución Tecnológica" que ha llegado a todos, de las cuales podemos sacar ventajas si sabemos usarlas. Hoy en día las redes sociales son las armas más populares y más al alcance que tenemos, pero ¿de qué clase de armas disponemos? La respuesta no está clara, pero sí sabemos que son armas muy potentes.

Es evidente que cada vez nuestras emociones están más condicionadas por las redes sociales; y esto lleva a una limitación de la maduración personal, ya que se pierde profundidad emocional a través de una pantalla. Los jóvenes creen que se puede conocer a una persona al completo sin conocerla físicamente. Esto es una gran falacia, no hay nada más emocional que una relación cara a cara ya que las emociones tienen un gran componente expresivo que se pierde a través de una red social, usando las redes como apoyo, no como sustitución, se llega a una vida emocional más plena.

En síntesis, los resultados nos muestran un uso elevado de internet $(29,8 \%)$ y de redes sociales $(21,7 \%)$, para hacer amigos. El uso de las redes sociales supone un cambio en los rasgos de personalidad siendo más atrevidos y menos extrovertidos. Del mismo modo, el uso de las redes sociales influye de manera positiva en las calificaciones obtenidas durante el curso escolar (63,5\%). Por otro lado, existe un distanciamiento familiar y de amistad en los adolescentes (85,5\%) aunque cuando tienen un problema grave derivado de su mal uso contactan con sus padres. Otros autores como Gracia Blanco, Vigo, Fernández Pérez, y Marcó Arbonés (2002) exponen que los principales problemas derivados del uso de las redes sociales son el distanciamiento de familiares o amigos, pérdida del tiempo de estudio. En cuanto a la personalidad que presentan a través del uso de las redes sociales observamos que ser sociales sigue siendo el principal con un 34,5\%, seguido de tranquilos con un $24 \%$, tímido (17\%), atrevido $(12,5 \%)$ y extrovertido $(12 \%)$. La alta frecuencia del uso de las redes sociales supone un cambio en las emociones de nuestros adolescentes, predominando las positivas.

No obstante, este trabajo presenta algunas limitaciones. En primer lugar, se trata de un estudio descriptivo que abarca una muestra pequeña de 200 adolescentes de la ciudad de Granada. Sería necesario contar con estudios que analicen muestras más amplias con patrones de uso específicos para cada rango de edad. En segundo lugar, dada la naturaleza descriptiva del estudio, los resultados hallados no demuestran los posibles indicios de riesgo y vulnerabilidad de forma específica en el uso de Internet y las redes sociales, para poder establecer pautas preventivas para desarrollar diversos comportamientos seguros a través de las redes sociales en nuestros jóvenes. Por último, sería conveniente analizar los resultados en función del sexo para ver el patrón de comportamiento entre hombres y mujeres, así como ampliar el estudio realizando un análisis cualitativo del tema estudiado.

Es por ello, que, como propuestas futuras de esta investigación, nos gustaría subrayar la posibilidad de canalizar una propuesta de formación e intervención en la era digital con adolescentes, padres y profesores para trabajar en contextos reales el adecuado uso de internet y redes sociales, así como la importancia de concienciar de la grave problemática que puede tener el uso incorrecto de las redes sociales en nuestra vida diaria.

\section{Referencias}

Beas, M., González, E., \& Salmerón, A. (2016). Estudio de las emociones en las consignas de cuadernos españoles. Curso 1964-1965. Revista de Estudios Sociales, 58, pp. 52-62. doi: $10.7440 /$ res58.2016.04.

Bisquerra, R. (2000). Educación emocionaly bienestar. Barcelona: Praxis.

Bisquerra, R. (2011). Educación emocional. Propuestas para educadores y familias. Bilbao: Desclée de Brower.

Boyns, D., \& Loprieno, D. (2014). Feeling through Presence: To - ward a Theory of Interaction 
Rituals and Parasociality in Online Social Works. En Benski, T., \& Fisher, E. (Eds.), Internet and Emotions (pp. 33-47). New York: Routledge.

Camps, V. (2012). El gobierno de las emociones. Barcelona: Herder Editorial.

Carrascal, L.F. (2016). Las emociones como formas de implicación en el mundo. El caso de la ira. Estudios De Filosofía, 53, pp. 81-101. doi: 10.17533/udea.ef.n53a05.

Casacuberta, D. (2000). Qué es una emoción. Barcelona: Crítica.

De Haro, J. J. (2010). Redes sociales para la educación. Madrid: Anaya.

Esquivel, L. (2001). El libro de las emociones. Valencia: Debolsillo.

Fowler, J.H., \& Christakis, N.A. (2008). Dynamic spread of happiness in a large social network: longitudinal analysis over 20 years in the Framingham Heart Study. [En línea]. Recuperado el 27 mayo de 2017 de: http://www.bmj.com/content/337/bmj.a2338.

García, M.C., Seco, J.A., \& Del Hoyo, M. (2013). La participación de los jóvenes en las redes sociales. Anàlisi: Quaderns de comunicació i cultura, 48, 95-110.

Garrido. L (2006). Apego, emoción y regulación emocional. Revista de Psicología, 38(3), 20-21. Recuperado de http://pepsic.bvsalud.org/pdf/rlp/v38n3/v38n3a04.pdf.

Gergen, K. J. (2015). El ser relacional: Más allá del Yo y de la Comunidad. Bilbao: Editorial Desclée de Brouwer.

Gracia Blanco, M., Vigo, M., Fernández, M., \& Marcó, M. (2002). Problemas conductuales relacionados con el uso de Internet: Un estudio exploratorio. Anales de Psicología, 18, pp. 273-292.

Küster D., \& Kappas, A. (2014). Measuring Emotions in Individuals and Internet Communities. En T. Benski, \& E. Fisher (Eds.) Internet and Emotions (pp. 48-61). New York: Routledge.

Lampe, C., Ellison, N., \& Steinfield, C. (2006). A Face (book) in the crowd: Social searching vs. social browsing. En Hinds, P., \& Martin, D. (Eds.) Proceedings of the 200620 th anniversary conference on Computer supported cooperative work (pp. 167-170). ACM. Recuperado de https://msu.edu/ nellison/lampe_et_al_2006.pdf.

Ledoux, J. (1996). El cerebro emocional. Barcelona: Planeta.

Llamas, F., \& Pagador, I. (2014). Estudio sobre las redes sociales y su implicación en la adolescencia. Salamanca: Ediciones Universidad de Salamanca.

Mahamud, K. (2012). Emociones y sentimientos: coordenadas históricas y multidisciplinares de un campo de estudio clave para las ciencias de la educación. Avances en supervisión educativa, 16, pp. 1 18. Recuperado de https://avances.adide.org/index.php/ase/article/view/500/340.

Molina, G., \& Toledo, K. (2014). Las redes sociales y su influencia en el comportamiento de los adolescentes, estudio a realizarse en cuatro colegios de la ciudad de Cuenca con los alumnos de Primer Año de Bacbillerato. Recuperado de: http://dspace.uazuay.edu.ec/handle/datos/3659.

Morgado, I. (2007). Emociones e inteligencia social. Barcelona: Ariel.

Oatley, K., \& Jenkins, J. (1992). Human emotions: Function and dysfunction. Anual review of Psychology, 43, pp. 55-85. doi: 10.1146/annurev.ps.43.020192.000415.

Orihuela, J. L. (2008). Internet: la hora de las redes sociales. Nueva Revista de Cultura, Politca y Arte, 119 , pp. 57-65.

Ponce, I. (2012). Monográfico: Redes Sociales. Madrid: Ministerio de educación, cultura y deporte. Recuperado en: http://recursostic.educacion.es/observatorio/web/es/internet/web-20/1043redes-sociales.

Real Academia Española. Emoción. [En línea]. [Consultado 26 Mayo 2017]. Recuperado de: http://dle.rae.es/?id=EjXP0mU.

Reeve, J. (1994). Motivación y Emoción. Madrid: McGrawHill.

Salas, J. (2016). ¿Qué quieres decir con un me gusta? El País. Recuperado de: http://tecnologia.elpais.com/tecnologia/2016/04/12/actualidad/1460484136_716565.htm.

Serrano-Puche, J. (2016). Internet y emociones : nuevas tendencias en un campo de investigación emergente. Revista Cientifica de Educomunicación, XXIV, 19-26. Recuperado de: file:///Users/erikagonzalezgarcia/Downloads/Dialnet-InternetYEmociones-5297319.pdf.

Stearns, P., \& Stearns, C. (1985). Etimilogy: clarifying the history of emotions and emotions standars. American Historical Review, 90, pp. 813-836. doi: 10.2307/1858841.

Svensson, J. (2014). Power, Identity, and Feelings in Digital Late Modernity: The Rationality of Reflexive Emotion Displays Online. En Benski, T., \& Fisher, E. (Eds.) Internet and Emotions (pp. 17-32). New York: Routledge. 
Tausiet, M., \& Amelang, J. (2009). Accidentes del alma: Las emociones en la Edad Moderna. Madrid: Abada. 